\title{
SOBRE EL TRATAMIENTO DE LOS SUPERLATIVOS EN EL DICCIONARIO DE AUTORIDADES
}

Resumen. El tratamiento de los superlativos en -ísimo en las primeras gramáticas y diccionarios del español presenta algunas características de interés que derivan de la peculiar historia de estos elementos. En el presente trabajo me propongo analizar el tratamiento que reciben en el Diccionario de Autoridades.

Palabras clave: superlativo, Diccionario de Autoridades, historia de la lexicografía.

\section{Introducción}

La historia de las formaciones superlativas (o, más bien, elativas) en -ísimo y su encaje en los tratados gramaticales del español constituye un caso atractivo y peculiar por diversas razones. Como es bien sabido, el sistema morfológico latino de positivo, comparativo y superlativo (altus, altior, altissimus) desapareció de las lenguas románicas y fue sustituido por procedimientos analíticos del estilo de alto, más alto, altísimo. La peculiaridad de la historia consiste, básicamente, en que el español conservó algunos comparativos existentes en latín (mayor, menor, etc.) $\mathrm{y}$, sobre todo a partir del siglo $\mathrm{XV}$, volvió a utilizar con cierta amplitud las formaciones superlativas en -ísimo, que se fueron reintegrando en el sistema y se convirtieron en un procedimiento morfológico productivo y, por tanto, aplicable en principio a cualquier positivo. Como consecuencia de todo ello, los gramáticos y lexicógrafos que se ocupan del español entre los siglos XV y XV III tienen que decidir si se puede hablar en esta lengua de comparativos más allá de los casos que proceden directamente del latín, si las formaciones en -ísimo (y asociadas) son realmente españolas o más bien latinismos y, por último, si los elementos del tipo muy alto son

\footnotetext{
* Universidade de Santiago de Compostela / Real Academia Española.
} 
equivalentes a los del tipo altísimo o, por el contrario, expresan significados próximos, pero distintos.

En un trabajo reciente (Rojo, 2019) me he ocupado de analizar el modo en que se plantean algunas de estas cuestiones a lo largo del proceso de preparación de la primera Gramática de la Academia, publicada en 1771. Me propongo aquí estudiar el tratamiento que estas formaciones reciben en el llamado Diccionario de autoridades (DAut), que constituye el primer enfrentamiento de los académicos fundadores con estas cuestiones y, además de todos los aspectos mencionados anteriormente, requiere la toma de decisiones acerca de si estos elementos, explicables y comprensibles como resultado de mecanismos morfológicos, deben figurar en el diccionario y, en caso afirmativo, en qué medida.

\section{La reintroducción de las formas en -ísimo}

Según la visión habitual, las formaciones en -ísimo se generalizan en español a partir del siglo XVI, lo cual explica su ausencia en la Gramática castellana de Nebrija, su rechazo explícito como procedimiento propio del español en Correas o incluso la parodia cervantina en el episodio de la dueña dolorida frente a, por ejemplo, las visiones más bien integradoras de Valdés, el Anónimo de 1555 o Ximénez Patón ( $c f$. Rojo, 2019, apdo. 3 y la bibliografía allí citada). El análisis de los datos existentes en los corpus diacrónicos que tenemos a nuestra disposición -el Corpus del español (CdEhist) y el Corpus diacrónico del español (CORDE)- permite perfilar mejor este proceso en algunos aspectos. Tal como se muestra en Rojo (2019), la utilización de estas formaciones es notable ya en la segunda mitad del siglo XV y aumenta sin cesar hasta la primera mitad del siglo XVIII, momento a partir del cual decrece en frecuencia hasta la actualidad. Tomando en consideración la frecuencia normalizada y también el número de lemas en que se pueden documentar estas formaciones, los datos correspondientes a la época en la que se gestan y publican el DAut y la primera Gramática de la Academia son los que aparecen en el cuadro 1, que muestran, con bastante claridad, que los primeros académicos están situados en un contexto de uso en el cual la formación en -ísimo está muy consolidada y ampliamente extendida, lo cual debe forzosamente influir en la consideración que les merece ${ }^{1}$.

${ }^{1}$ La frecuencia normalizada es, en este caso, el número medio de apariciones por cada millón de palabras. A este dato, que es la frecuencia de uso, es importante añadir la 
Cuadro 1. Número de lemas distintos y suma de frecuencias normalizadas (casos por millón) de las formas en -ísimo en diferentes tramos del CORDE

\begin{tabular}{ccc}
\hline & Número de lemas & $\begin{array}{c}\text { Suma de frecuencias } \\
\text { normalizadas }\end{array}$ \\
$1651-1700$ & 462 & 981,39 \\
$1701-1750$ & 574 & 1409,64 \\
$1751-1800$ & 530 & 996,38 \\
\hline
\end{tabular}

Fuente: CORDE. Elaboración propia (cf. Rojo, 2019: cuadro 2).

\section{Los superlativos en el Diccionario de Autoridades}

La primera relación de los académicos fundadores con las cuestiones relacionadas con el superlativo se produce, como es lógico, en el DAut y se mueve en las dos direcciones esperables en la lexicografía del siglo XVIII. Por una parte, las definiciones de las acepciones gramaticales de voces como grado, positivo, comparativo y superlativo muestran el modo en que concebían estos conceptos gramaticales. Pero tan importante como eso, o quizá incluso más en este caso, resulta la relación de superlativos incluidos en el DAut y el modo en que se realiza su caracterización.

En el primero de los dos aspectos, surge la dualidad de consideración mencionada, como señaló ya Val Álvaro (1992: 59 y sigs.). En la definición de grado (en su acepción gramatical) dicen que

es el excesso en la significación de las voces, respecto de la voz possitiva de donde nacen, quales son comparatívos y superlatívos. Conocemos el comparatívo en estas partículas Mas y que: como Qué cosa más blanda que la ola? Y usaremos del superlatívo grado, conociéndole por estos romances Mui, o Más de (DAut, s.v. grado).

Es decir, «se trata de una propiedad definida en términos semánticos y expresable sintácticamente», en palabras de Val Álvaro (1992: 60). En cambio, el comparativo es «el nombre cuyo significado aumenta el del Positivo, con relación a otro, como Mejor respecto de Bueno. Peor respecto de Malo» (DAut, s.v. comparativo) y el superlativo «[en] la Gramática se aplica al nombre cuyo significado pone à la cosa en grado, y estima, ò mui alta, ò mui baxa: como Malissimo, bonissimo, \&c.» (DAut, s.v. superlativo). Como se puede apreciar, «este tipo de adjetivos son considerados como

frecuencia de inventario, que consiste en el número de elementos distintos que presentan esta formación. Para detalles sobre los dos conceptos, cf. Rojo (2011). 
términos semánticamente distinguidos por su variación morfológica» (Val Álvaro, 1992: 60). No puede ser casual el hecho de que en todas estas entradas, así como en la correspondiente a positivo, aparezca entre las autoridades una cita de Ximénez Patón, completa únicamente en la del superlativo:

Los tres grados de nombres, positiuos, comparatiuos y superlatiuos los ay en cierta manera, porque no guardan la formación que entre los latinos, si no es los superlatiuos que, o vienen dellos, o les imitan, como santíssimo, boníssimo. Lo que tiene de su idioma es suplir con dos adueruios estos grados, el positivo y más, en las comparaciones, como más sabio, más santo; y muy en los superlatiuos, como muy ábil, muy fiel. Ta[m]bién a tomado algunos comparatiuos de los latinos, como mejor, peor, menor, mayor. La construción del comparatiuo es la conjunción que, como más bueno que otro, o mejor que otro, peor que tú, o más malo q[ue] tú, \&c (Ximénez Patón, [1614] 1965: 98).

El segundo aspecto, de mayor interés para nuestro propósito, radica en cómo se plantea la conveniencia de introducir formas en -ísimo y similares en el diccionario, los criterios que se siguen para su inclusión y el resultado de todo ello, esto es, qué número de superlativos sintéticos entran en la obra y cómo se estructura su definición. Los redactores del DAut son perfectamente conscientes de las dificultades que se presentan en el caso de zonas como esta en las que confluyen lo léxico y lo gramatical. En el prólogo de la obra señalan que, dado que este tipo de voces (derivadas y compuestas) pueden formarse a partir de todas las bases adecuadas, han optado por incorporar, en el caso de los diminutivos, aumentativos y superlativos, únicamente los más usados y registrados en los autores más destacados:

Es fecundissima esta Léngua en los diminutivos, y aumentativos, cuyas derivaciones son várias $[\ldots]$ y de este genero se pueden sacar diminutivos, y aumentativos de casi todas las voces, como igualmente los superlativos, porque en esto es la Léngua mui voluntária, cuya expressión sería sumamente difusa y penosa, y en este Diccionario se ponen solo los mas usados, y que se hallan autorizados por los Escritores escogidos (DAut, prólogo: vi).

En efecto, esa parece ser la técnica que adoptan a lo largo de toda la obra, con algunas peculiaridades a las que aludiré posteriormente. Como ya indicó Freixas (2010: 262) la forma de definir los superlativos es uno de los asuntos sometidos a revisión en 1731, cuando, publicados ya los dos primeros tomos de la obra, la Academia decide encargar a dos revisores (Lorenzo Folch de Cardona y Tomás Pascual de Azpeitia) que estudien lo ya impreso para que lo pendiente resulte más homogéneo. En este punto concreto, en el acta correspondiente al 7 de junio de 1731 se 
indica que Folch de Cardona continuó leyendo su documento de "reparos al Diccionario" y, entre otros temas, se refiere a

las variedades que encuentra en la explicación de los superlativos, que en unos se dice Adjetivo superlativo y en otros no más que Adjetivo. en unos se expresa el positivo de que se formaron y en otros no, y unos se explican ù definen a lo largo y otros solam. ${ }^{\text {te }}$ con la voz mui, y el mismo positivo, y pretenden los ss $^{\text {res }}$ Revisores regla fixa para lo de adelante: sobre que se pasó a votar y quedó resuelto se ponga siempre Adjetivo superlativo de tal nombre y que la explicación se reduzca solo à la voz mui y el positivo, como: Decentísimo. Adjetivo superlativo de decente. Mui decente (Actas RAE, 7/6/1731. Cf. también Freixas, 2010, 262, nota 91)².

El análisis de las primeras entradas de este tipo incluidas en el DAut muestra estas divergencias con toda claridad: abatidissimo es caracterizado como "adj. superl." y definido como "sumamente humillado y rendido"; de abrasadissimo, que es la siguiente de este tipo, se dice que es "superl." y se define como "muy abrasado y quemado"; abstinentissimo es "superl. de Abstinente" que significa "muy abstinente y sumamente parco"3. Sin embargo, a partir de la letra D todos estos elementos se ajustan a lo convenido y, salvo algunos casos marginales como el mencionado en la nota 3, parece que esto se mantiene así hasta el final de la obra.

La versión digitalizada del DAut que existe en la página electrónica de la $\mathrm{RAE}^{4}$ permite hacer un análisis cómodo y rápido del grado en que se ejecuta la decisión de incluir solo los casos más frecuentes y documentados en los mejores autores. Como primera aproximación, hay 594 entradas en las que el elemento es caracterizado como "superl."

${ }^{2}$ Agradezco a Covadonga de Quintana, directora del Archivo de la RAE, la ayuda que me ha prestado para la consulta de las actas de los plenos y los dictámenes e informes que figuran entre los documentos de preparación de la primera edición de la GRAE.

3 Para las recuperaciones automáticas del contenido del DAut tienen también importancia factores como las oscilaciones en las abreviaturas (como "superlat." en antiquissimo), empleo de la palabra completa (en una de las entradas de altissimo), la indicación de superlativo en la primera de las entradas correspondientes a un lema y el empleo de otras fórmulas en las siguientes (como en acomodadissimo). A mayor distancia se sitúan recursos como, por ejemplo, el usado en infinitissimo de la que se dice "adj. Compuesto de infinito, en significación de innumerable. Voz solamente del estilo familiar o jocoso" o en abstinentissimamente, que definen como "adv. superl. Con suma y rigurosissima abstinencia y privacion estrecha de qualquier gusto y deléite sensual, y lo mismo que estrechissima y mui parcamente" y justifican con una cita de Fr. Luis de Granada. Todos estos casos marginales complican la realización automática de recuentos, como se indica en lo que sigue.

${ }^{4}$ Para las características de este recurso, $c f$. Rojo (2014).

${ }^{5}$ Más dos de "superlat." (antiquissimo y caprichosissimamente). 
1,4\% de los 42368 lemas contenidos en la obra ${ }^{6}$. Como se indica en el párrafo anterior, esta abreviatura no es la utilizada en todos los casos, de modo que resulta más fiable recurrir a la forma de la palabra, con lo que se obtienen los resultados que aparecen en el cuadro 2:

Cuadro 2. Frecuencia de diferentes tipos de superlativo en el DAut.

\begin{tabular}{cc}
\hline Forma & Frec. \\
\hline -issimo & 493 \\
-errimo & 7 \\
-issimamente & 113 \\
-limo (facílimo, dificílimo) & 2 \\
\hline Total & 615 \\
\hline
\end{tabular}

Fuente: RAE (DAut). Elaboración propia.

Es fácil prever la aparición de discrepancias en los resultados obtenidos en la aplicación de los rasgos mencionados en el prólogo (frecuencia de uso y documentación en los autores seleccionados) para la inclusión de elementos que, como los superlativos, aumentativos y diminutivos, no aparecen habitualmente en los diccionarios, precisamente por su 306 adscripción al componente morfológico. El primer modo de intentar saber en qué medida los primeros académicos mantienen una cierta congruencia en la aplicación de estos principios consiste en comprobar la proporción de superlativos que figuran en los seis tomos de la obra. Los datos del cuadro 3 muestran que, en efecto, hay discrepancias de entidad entre el porcentaje total $(1,45 \%)$ con respecto a los extremos, representados por el reducido $1,15 \%$ del primer tomo y el mucho más abultado $1,8 \%$ del tercero.

${ }^{6}$ Soy consciente de la discrepancia que se crea al poner en relación el número de entradas que poseen la indicación 'superl.', obtenido de la consulta de la aplicación, con el número de lemas contenidos en la obra, procedentes del listado que figura en la misma página. Como es bien sabido, la organización del DA hace figurar en párrafos distintos cada una de las acepciones, expresiones complejas, locuciones, refranes y otras expresiones asociadas a cada lema, normalmente bien diferenciadas mediante recursos tipográficos, de modo que forzosamente hay más entradas que lemas. La aplicación de consulta que funciona en la página de la RAE presenta los resultados devueltos como 'número de lemas', pero es fácil comprobar que se trata, en realidad, de número de entradas. En este caso concreto, poner en relación las 594 entradas con la marca 'superl.' con las 59248 entradas qe contiene la obra daría una imagen distorsionada del peso real de este componente. Aunque sin duda introduce un cierto desajuste, el efecto de comparar las entradas con 'superl.' con el número total de lemas es mucho menor, puesto que son pocos los casos en los que un lema con esta marca contiene más de una entrada. Para más detalles sobre número de entradas y lemas en el DAut, cf. Rojo (2014: 145). 
Cuadro 3. Número de superlativos, número de lemas y porcentaje de superlativos en los tomos del DAut.

\begin{tabular}{cccc}
\hline & Lemas superlativos & Total lemas & Porcentaje \\
A-B & 87 & 7567 & 1,15 \\
C & 81 & 5218 & 1,55 \\
D-F & 160 & 8888 & 1,80 \\
G-N & 105 & 7434 & 1,41 \\
O-R & 100 & 6877 & 1,45 \\
S-Z & 82 & 6373 & 1,29 \\
TOTAL & 615 & 42357 & 1,45 \\
\hline
\end{tabular}

Fuente: RAE (DAut) y Rojo, 2014. Elaboración propia.

Una vía más interesante para tratar de evaluar la medida en que los criterios mencionados son aplicados realmente consiste en comparar lo incluido en el DAut con los datos procedentes de los textos de la época. Las listas de frecuencia del CORDE (http://www.rae.es/recursos/bancode-datos/corde) para los períodos 1651-1700 y 1701-1750, recogen 462 y 574 lemas, respectivamente, con un total de 694 lemas distintos para estos cien años. Es decir, una cifra bastante similar a la reflejada en el DAut.

Naturalmente, el simple contraste de las cifras no garantiza la congruencia de las dos aproximaciones, de modo que he comparado algunos fragmentos de las dos listas. En los cien lemas con superlativo en -ísimo más frecuentes en las listas de frecuencia del CORDE correspondientes al período 1701-1750, con frecuencias normalizadas superiores o iguales a 2,723 casos por millón, detecto únicamente 8 ausencias (las correspondientes a diverso, tenue, amante, vasto, distinto, verdadero, extraordinario y recto). Entre los cincuenta lemas situados en una zona media (entre los puestos 201 y 250, con frecuencias normalizadas comprendidas entre 0,961 y 0,64), hay 19 superlativos que aparecen en los textos y no figuran en el DAut, lo cual supone que la obra contiene únicamente el $60 \%$ de los superlativos de ese tramo detectados en los textos. En los cincuenta lemas situados en la zona más baja (entre los puestos 501 y 550, con frecuencias normalizadas equivalentes a 0,16 ), he detectado 25 ausencias. En general, parecen unos porcentajes congruentes $\mathrm{y}$, teniendo en cuenta las circunstancias que pesan en la lexicografía de la época sobre la selección de lemas, bastante aceptables (8\%, 40\% y 50\%, respectivamente, en los tramos alto, medio y bajo). 
La perspectiva contraria da también resultados positivos sobre el modo de trabajar de los primeros académicos. Utilizando la lista de lemas, he extraído los veinte primeros superlativos en -ísimo de cada tomo y he comprobado si están documentados en las listas de formas del CORDE entre 1601 y 1750. De las 120 voces comprobadas, únicamente 9 no figuran en ese período del CORDE, pero todas ellas salvo 3 llevan una cita textual que abona su uso, lo cual inclina a pensar que la discrepancia se debe más al carácter forzosamente limitado del CORDE que a la inconsistencia del método aplicado por los primeros académicos ${ }^{7}$.

Cuadro 4. Algunos superlativos presentes en el DAut no documentados en el CORDE entre 1601 y 1750

\begin{tabular}{lcc}
\hline \multicolumn{1}{c}{ Forma } & Citas en DAut & Observaciones (en DAut) \\
\hline abstractíssimo & 1 & Voz de poco uso \\
achacosíssimo & 0 & \\
afectíssimo & 0 & \\
alabadíssimo & 1 & \\
calamitosíssimo & 1 & \\
demasiadíssimo & 0 & \\
desasidíssimo & 1 & \\
desagradecidíssimo & 1 & \\
ganosíssimo & 1 & \\
\hline
\end{tabular}

Fuente: RAE (DAut y CORDE). Elaboración propia.

Veamos ahora en qué medida se respeta el segundo de los factores mencionados en el prólogo: el referente al uso de estas formas en los 'escritores escogidos'. Mediante un procedimiento similar al empleado en ocasiones anteriores ( $c f$. Rojo, 2014 y 2016) he extraído las citas textuales que figuran en las entradas correspondientes a las formas en -ísimo. El resultado es un inventario constituido por un conjunto de 146 autores ( $u$ obras en caso de que sean citadas directamente) que abonan con sus textos estas entradas. La relación de los 20 más utilizados aparece en el cuadro 5:

${ }^{7}$ Resulta destacable, por la acumulación de procesos, el caso de superabundantissimo, recogido en el DAut, definido, de acuerdo con el sistema aprobado en 1731, como "adj. superl. Mui superabundante" y autorizado con otra cita de Fray Luis de Granada. El CORDE no contiene el texto utilizado en el DAut, pero sí una aparición previa de la palabra en un sermón de fray Alonso de Cabrera fechado en 1598. De todas formas, hay que tener en cuenta que la existencia de una cita textual en el DAut no garantiza el cumplimiento del compromiso de inclusión de los superlativos 'más frecuentes'. 
Cuadro 5. Frecuencias de los veinte autores u obras más citados en las entradas del DAut de formas en -ísimo

\begin{tabular}{llll}
\hline CERV. & 32 & M. AGRED. & 16 \\
RIBAD. & 27 & ALCAZ. & 15 \\
BAREN & 24 & HORTENS. & 15 \\
PUENT. & 23 & ILLESC. & 15 \\
ACOST. & 19 & MANER. & 15 \\
FUENM. & 19 & QUEV. & 15 \\
OV. & 19 & CORN. & 14 \\
NIEREMB. & 18 & COMEND. & 13 \\
PELLIC. & 18 & FR. L. DE GRAN. & 13 \\
PALAF. & 17 & MEND. & 13 \\
\hline
\end{tabular}

Fuente: RAE (DAut y CORDE). Elaboración propia.

De ellos, solo 5 no figuran entre los 40 más citados en la totalidad del DAut (BAREN., PUENT., PALAF., ILLESC. y CORN.), de modo que es posible concluir que, al menos en lo que se refiere al segmento más importante desde el punto de vista cuantitativo, las citas de las entradas correspondientes a los superlativos se ajustan a los principios generales utilizados en la obra ${ }^{8}$.

\section{Final}

Entre 1740 y 1747, además de publicar la Ortografía, los académicos se dedican con intensidad a la nueva edición del diccionario y también a preparar la futura Gramática. Esta segunda tarea se realiza en esta época fundamentalmente mediante la discusión de cuestiones que Casani había extraído previamente de los proyectos presentados poco tiempo antes por Francisco de Angulo e Ignacio de Ceballos, con notas adicionales de Carlos de la Reguera9. Una de las cuestiones seleccionadas por Casani fue «que sea positivo, comparativo y superlativo, si los ay en $\mathrm{n}^{\text {ra }}$ lengua y su

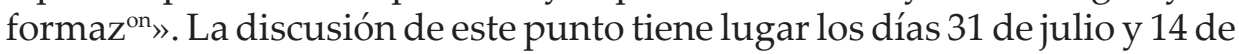
agosto de 1742 y la documentación conservada permite ver que Ignacio de Luzán es uno de los participantes más activos y que, en concreto,

${ }^{8}$ Para todo lo relacionado con las citas, las abreviaturas utilizadas y las dificultades existentes para su manejo, $c f$. Rojo (2014 y 2016).

${ }^{9}$ Para detalles sobre este proceso, vid. Rojo (2001: 19 y sigs. y la bibliografía allí citada). 
mantiene que los procedimientos del tipo grandísimo y muy grande no son equivalentes, por lo que hay que reconocer la existencia de cuatro grados: «Positivo Comparativo, superlativo inferior, o impropio con el adverbio Muy; y superlativo proprio, que es comunmente el acabado en ssimo» (Luzán, dictamen presentado el 14/8/1742, FRAE 2/3/11) ${ }^{10}$.

Quizá sea esta la razón de que en la segunda edición del DAut (1770) y en la primera del DRAE (1780) la fórmula definitoria de los superlativos se haya cambiado y ahora sea ya del tipo «ABUNDANTÍSIMO, MA. adj. sup. de ABUNDANTE» (DRAE 1780, s.v.). Así se mantiene durante los cien años siguientes, hasta la duodécima edición del DRAE $(1884)^{11}$, en la que desaparecen del lemario todos los superlativos regulares. Las Reglas para la corrección y aumento del Diccionario vulgar editadas en 1870 indican lo siguiente:

(Voces derivadas y voces compuestas) Se incluirán todas las derivadas y compuestas de uso autorizado, excepto los superlativos regulares, cuidándose especialmente de no omitir ninguna de las que pueden dar lugar á dudas respecto de su verdadero sentido ú ofrezcan alguna irregularidad ó circunstancia notable en su formacion $(\mathrm{RAE}, 1870: 5)^{12}$.

\section{Bibliografía}

CLAVERÍA NAD A L, G. (2016). De vacunar a dictaminar: la lexicografía académica decimonónica y el neologismo. Madrid: Iberoamericana Vervuert.

FREIXAS ALÁS, M. (2010). Planta y método del Diccionario de Autoridades. Orígenes de la técnica lexicográfica de la Real Academia Española (1713-1739). Anexo 14 de la Revista de Lexicografía. La Coruña: Universidade da Coruña.

GARRIGA ESCRIBANO, C. (2001). "Sobre el diccionario académico: la 12. ${ }^{\text {a }}$ ed. (1884)», en A. M. MEDINA GUERRA (coord.), Estudios de lexicografía diacrónica del español. (V centenario del Vocabularium Ecclesiasticum de Rodrigo Fernández de Santaella). Málaga: Universidad de Málaga, 261-315.

10 Para detalles sobre esta documentación, $c f$. Rojo (2001, 2018). La importante labor de análisis de estos materiales que se está llevando a cabo en el Archivo de la RAE, dirigido por Covadonga de Quintana desde 2015, permite ya la consulta ordenada de todos estos documentos. Para su inventario y sistema de referencias, $c f$. Quintana (2017).

${ }^{11}$ Sobre las características e importancia de esta edición, $c f$. , entre otros, Garriga Escribano (2001) y Clavería (2016: 172 y sigs.)

12 Es innovación clara con respecto a la edición de 1869 en la que se dice: «Se incluirán todas las voces derivadas y compuestas de más frecuente uso, sin omitir ninguna de las que usadas o no con frecuencia, puedan dar lugar a dudas $[\ldots] »$. 
QUINTANA, C. DE (2017). Inventario de los documentos de trabajo para la Gramática de la Real Academia Española (1740-1767) [en línea] <http://archivo.rae.es/ index.php/dictamenes-disertaciones-y-votos-particulares-sobre-gramatica-s -xviii>, fecha de consulta: 10 de abril de 2019.

REAL ACADEMIA ESPAÑOLA (1869). Reglas para la corrección y aumento del diccionario vulgar. Madrid.

REAL ACADEMIA ESPAÑOLA (1870). Reglas para la corrección y aumento del diccionario vulgar. Madrid.

ROJO, G. (2001). El lugar de la sintaxis en las primeras gramáticas de la Academia. Madrid: RAE.

ROJO, G. (2011). «Frecuencia de inventario y frecuencia de uso», Revista española de lingüística, 41/1, 5-43.

ROJO, G. (2014). «Análisis cuantitativo de las citas del Diccionario de Autoridades», BRAE, XCIV, 137-196.

ROJO, G. (2016). «Cuantificación de las citas textuales del Diccionario de Autoridades» [en línea] <http://gramatica.usc.es/persoas/guillermo.rojo>, fecha de consulta: 10 de abril de 2019.

ROJO, G. (2018). «Sobre el tratamiento de los superlativos en la preparación de la primera Gramática de la Academia (1771)». En prensa.

VAL ÁLVARO, J. F. (1992). Ideas gramaticales en el Diccionario de Autoridades. Madrid: Arco.

XIMÉNEZ PATÓN, B. ([1614] 1965). Instituciones de la Gramática española. Cito por la edición de Antonio Quilis y Juan Manuel Rozas. Madrid: CSIC.

Corpus textuales y otros recursos electrónicos mencionados en el texto

CdEhist - Corpus del español: Género / histórico [en línea] <https://www.corpusdelespanol.org/hist-gen>.

CORDE - Corpus diacrónico del español [en línea] <http://corpus.rae.es/cordenet. html>.

DAut - Diccionario de Autoridades (1726-1739) [en línea] <http://web.frl.es/DA. html>.

Real Academia Española. Libros de actas de las sesiones plenarias.

Real Academia Española. Documentos de trabajo elaborados en el proceso de preparación de la primera Gramática de la Real Academia Española (cf. Quintana, 2017). 